\title{
Early Detection of Suicide Using Big-Data Analytics in Real Time
}

\author{
Hardik A. Patel, Cheng-Yuan Hsieh \\ Knowledge Systems Institute
}

Abstract-This paper presents an application, wherein predictive analysis is used to churn social media comments and posts for identifying individuals on these sites susceptible to suicidal thoughts and tendencies. So an early intervention could be made saving lives of such individuals. Also presented is a model wherein a branch of big data analytics and sentimental analytics has been used to identify profiles of users with suicidal posts.

Index Terms-Big data, Real-time, Predictive analysis .

\section{INTRODUCTION}

This application is meant to be used by suicide prevention agencies. The growth of social media has presented a new set of challenges and opportunities in the field of suicide prevention. Studies have shown that many people have presented their feeling related to depression on social media [1]. A timely intervention could save lives of people who might have not given a thought of getting help themselves by going to counseling services offered by these agencies. American Foundation for Suicide Prevention (AFSP) has a webpage dedicated to warnings of suicide [2], or indications where a person might be showing suicidal tendencies. Research has shown that suicidal ideation or self-injurious behavior is an early warning of suicide [3]. American Association of Suicidology (Suicidology.org), lists vocalizing suicidal thoughts as one of the primary warning indicators for suicide. This application will provide assistance to organizations by fetching information from social media websites like Facebook, Twitter etc., in real time when posts and/ or comments exhibiting such tendencies are made.

\section{RESEARCH MEthodology}

The impact of media on suicidal behavior seems to be most likely when a method of suicide is specified in detail. Sometimes newspapers or other media outlets like radio, television or the internet publish suicide stories or reports in details as to how it happened and what methods were used in the suicide. Suicide prevention agencies, both federal state and private charities, have been very active in helping individuals with depression and suicidal behavior. The main drawback of conventional systems is timely intervention. All of the conventional systems relied primarily to individuals seeking out help for themselves or someone they know. All other cases where the primary warning signs are not detected or the individual is hesitant to seek out help, these agencies with all their excellent resources would not be able to provide any help. The application will identify such posts based upon certain data sets. These data sets comprise of certain words/phrases which help the application identify such profiles by pointing to the associated analysis would provide suicide prevention agencies

a list of such subjects who might need help/intervention to combat through their depression and anxieties associated with suicide victims.

\section{SYSTEM OVERVIEW}

The system has a multi-tier Architecture and includes web server, a PHP MySQL server, a Linux server hosted on Digital Ocean cloud ecosystem and a MongoDB [4] database for storing and retrieving the tweeter feed in JSON format. The live twitter feeds are fed from the MongoDB database on the Linux server provided by Digital Ocean Cloud infrastructure provider[5]. Sentiment analysis on the twitter feeds takes place in the Linux server and the resulting output is stored in the MongoDB database in JSON format. The web application accesses this information and populates it on the Home Screen. An agent can see twitter feeds which are captured in real-time, where as a supervisor can see the tweets flagged by the Agents. Then a timely intervention could be issued.

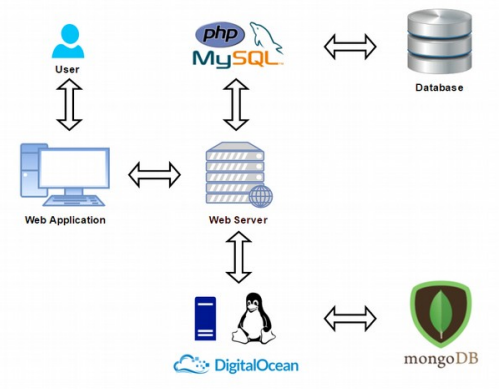

\section{CONCLUSION}

The is a tool for the suicide prevention agencies who can use it to do predictive analysis using big data, in identifying individuals suffering from suicidal tendencies, thereby helping them to make a timely intervention and saving lives.

$$
\mathrm{V} \text {. References }
$$

[1] K. M. M. N. M. E.-F. M. \&. S. M. Becker, "Parasuicide online: Can suicide websites trigger suicidal behaviour in predisposed adolescents?," Nordic Journal of Psychiatry,, vol. 58, pp. 111-4, 2004.

[2] "American Foundation for Suicide Prevention,"[Accessed $\left.\begin{array}{lll}11 & 01 & 2016\end{array}\right]$ https://www.afsp.org/understandingsuicide/suicide-warning-signs..

[3] R. D. Goldney, "Suicide Prevention," Oxford University Press, 2008, p. 11.

[4] Tutorials Point, "Mongo DB," [Accessed 09 March 2016] http://www.tutorialspoint.com/mongodb/mongodb_overvi ew.htm.

[5] Databricks, "Apache Spark," Databricks, [Accessed 31 January 2016] https://databricks.com/spark/about. 